\title{
Morfometría Geométrica del Corazón de Hyla plicata a Través de un Gradiente Altitudinal en el Eje Neovolcánico Mexicano
}

\author{
Geometric Morphometric of the Heart in Hyla plicata Through \\ an Altitudinal Gradient in the Mexican Neovolcanic Axe
}

Rios-Rodas, L."; Rodríguez-Romero, F. de J."; Velázquez-Rodríguez, A. S." \& Hernández-Franyutti, A. A.**

RIOS-RODAS, L.; RODRÍGUEZ-ROMERO, F. J.; VELÁZQUEZ-RODRÍGUEZ, A. S. \& HERNÁNDEZ-FRANYUTTI, A. A. Morfometría geométrica del corazón de Hyla plicata a través de un gradiente altitudinal en el eje Neovolcánico Mexicano. Int. J. Morphol., 31(3):905-910, 2013.

RESUMEN: Se ha descrito que el medio aéreo representa menos problemas a los organismos en relación con la hipoxia a excepción de circunstancias especiales, como la exposición a grandes alturas o las madrigueras. El ambiente de gran altitud es desafiante en diversos sentidos, dado que existen factores bióticos y abióticos que pueden interactuar para determinar la distribución y sobrevivencia de los organismos. Para conocer estos aspectos, la biología se apoya en la ecomorfología, área que ayuda a definir las diferencias morfológicas con respecto al cambio del hábitat y en la morfometría geométrica que permite analizar estadísticamente esos cambios. En el presente estudio, se evaluaron diferentes poblaciones de la rana Hyla plicata que es una de las especies que se distribuyen a mayor altitud en México, (1500 m hasta los 3700 m). Por lo que el objetivo de este estudio, fue analizar la morfología del corazón en Hyla plicata a través de un gradiente altitudinal. Para ello se colectaron organismos en cuatro localidades, una de ellas fue en La Cima (Distrito Federal a 3016 m), y tres ubicadas en el Estado de México: Buenavista (3200 m), El Cerrillo (2632 m) y San Juan Atezcapan (1860 m). Los organismos colectados se disectaron para extraer el corazón con la finalidad de digitalizarlos mediante fotografías, y analizarlos con puntos geométricos y de esta forma evaluar la forma del órgano. Los resultados indican que las localidades de mayor altitud presentan aurículas y ventrículos más ensanchados, lo que señala que esta estructura evolucionó y se adaptó para efectuar un bombeo más eficaz de la sangre y posteriormente dirigirla hacia los diferentes tejidos del cuerpo. Esta adaptación en la morfología del corazón, permitió que Hyla plicata se adaptara a los ambientes de alta montaña como se ha visto en otros vertebrados que habitan zonas de mayor altitud.

PALABRAS CLAVE: Morfometría geométrica; Adaptación; Gradiente altitudinal; Hyla plicata; Corazón.

\section{INTRODUCCIÓN}

El contenido de oxígeno en el aire es más abundante que en el agua, por lo que el medio aéreo presenta menos problemas relacionados con la hipoxia, en comparación con el medio acuático; la hipoxia en los vertebrados terrestres se encuentra en dos entornos naturales, uno con el incremento en la altitud y otro en las madrigueras subterráneas (Hillman et al., 2009; Jackson, 2007). El ambiente de alta montaña es desafiante en muchos sentidos, ya que es frio, ventoso, deshidratante, con variaciones significativas en la temperatura, presentan una elevada exposición a la radiación ultravioleta y un descenso significativo en la presión parcial de oxígeno, y en su conjunto, pueden interactuar e influir en la distribución y sobrevivencia de los organismos e incluso llevarlos un estado de hipoxia, situación que puede repercutir en la reproducción de los organismos. Estas características extremas afectan tanto a los animales terrestres como a los acuáticos y puede llevarlos a formar adaptaciones para hacer frente a estas condiciones (Hillman et al.; Hill, 2007; Hill et al., 2008; Jackson; Campbell et al., 2001).

Otros estudios han abordado el efecto que tiene la baja disponibilidad de oxígeno en los organismos, tanto en condiciones controladas de laboratorio o en su ambiente natural (Ruiz-Valencia, 2010; Scott et al., 2009; Marques et al., 2008; Moyes \& Schulte, 2008; Valenzuela et al., 2002; Campbell; Alerstam, 1990), sin embargo, sólo algunos estudios se han realizado en anfibios (Méndez et al., 2004; Ruiz et al.,1989; Hutchison, 1982; Ruiz et al., 1983; Hutchison et al., 1976) pero limitados son aquellos que observan si hay un efecto de la hipoxia sobre la morfología externa del corazón.

\footnotetext{
* Facultad de Ciencias, Universidad Autónoma del Estado de México, Toluca, México.

** División Académica de Ciencias Biológicas Universidad Juárez Autónoma de Tabasco, Tabasco, México.
} 
Para probar esta hipótesis en un ambiente hipóxico, se estudió a la especie Hyla plicata, que es una rana endémica del centro de México, de hábitos semiacuáticos y se encuentra principalmente asociada a las orillas de las charcas temporales durante la época de la reproducción; además de ser el hílido que se distribuye a mayor altitud (1500 $3700 \mathrm{~m}$ ) en el eje Neovolcánico de México (Duellman, 1970). Por lo tanto, el objetivo de este estudio fue analizar la morfología del corazón en poblaciones que habitan diferente altitud para conocer la evolución y adaptación de este órgano en poblaciones que habitan ambientes extremos de alta montaña.

\section{MATERIAL Y MÉTODO}

Áreas de estudio.Se colectaron individuos de la especie Hyla plicata en poblaciones que habitan a diferente altitud, La Cima en el Distrito Federal (3018 m), Buenavista (3216 m), El Cerrillo Piedras Blancas (2632 m) y San Juan Atezcapan (1860 m) las últimas tres ubicadas en el Estado de México (Fig. 1).
Morfometría Geométrica. Se disecaron, en promedio 10 organismos de cada localidad para extraer el corazón. Posteriormente se obtuvieron fotografías digitales de la vista ventral del corazón, con una cámara digital (Sony DSLRa350, a una resolución de 14 megapixeles), colocada a una distancia de $20 \mathrm{~cm}$ del corazón y con una escala métrica de referencia al milímetro más cercano $(0,1 \mathrm{~mm})$.

Dado que el corazón posee una forma curva, no se pueden utilizar puntos homólogos, por lo que se optó por usar el método de los semilandmarks (Zelditch et al., 2004), que permiten referir una serie de puntos a lo largo de la curva y que pueden analizar la variación en la forma como los landmarks verdaderos. Una característica de los semilandmarks es que deben ubicarse en intervalos iguales a lo largo de la curva, por lo que, antes de la digitalización, se deben colocar peines y/o abanicos, de manera que se pueda cubrir toda la forma externa del corazón, para ello, se utiliza el módulo MakeFan del paquete estadístico IMP (Integrated Morphometrics Package). Para trazar los peines y abanicos es necesario ubicar puntos de referencia, que pueden ser al menos dos landmarks verdaderos; para la presente investigación, estos puntos se colocaron en la unión del ventrículo y las aurículas (Fig. 2).

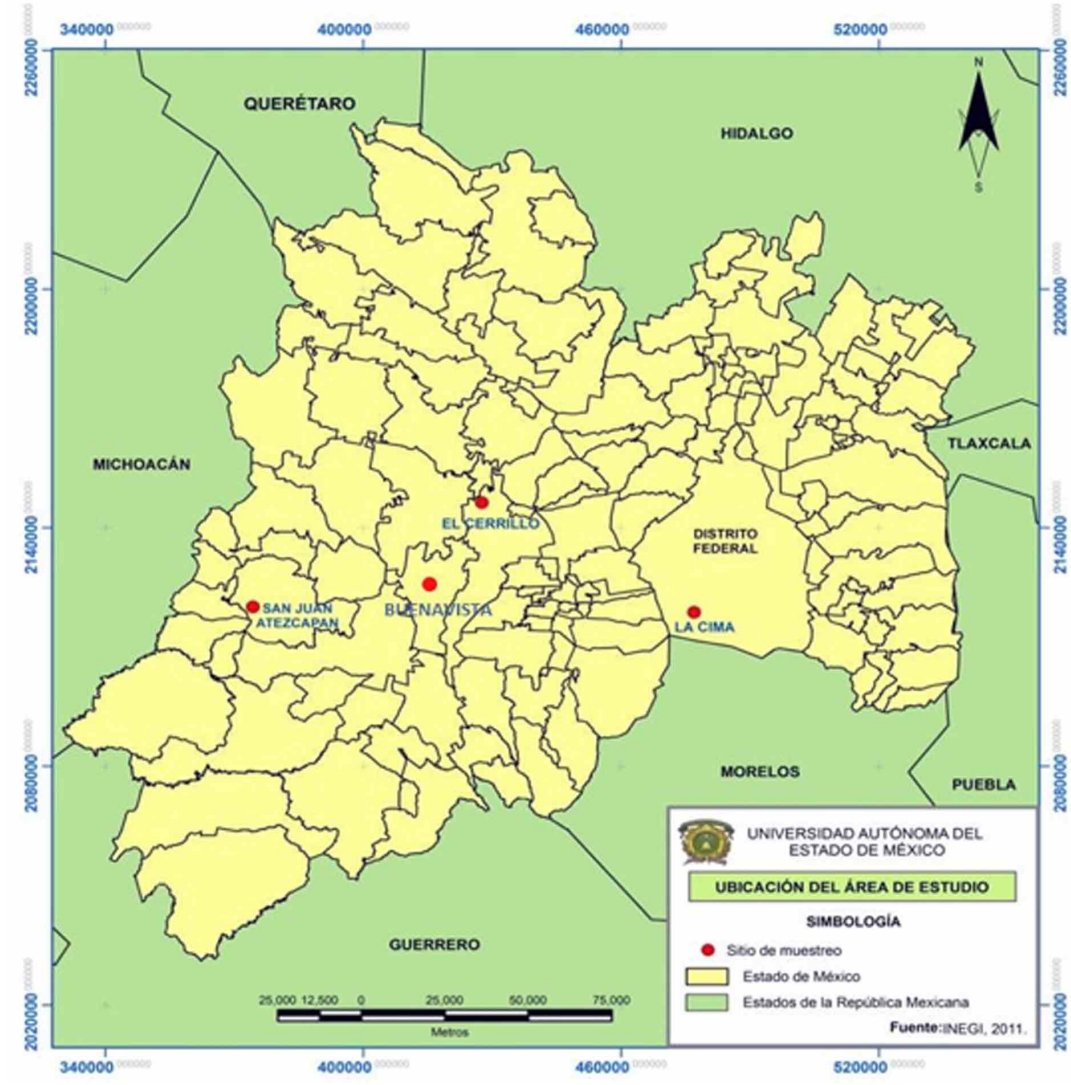

Fig. 1. Ubicación de las poblaciones analizadas.

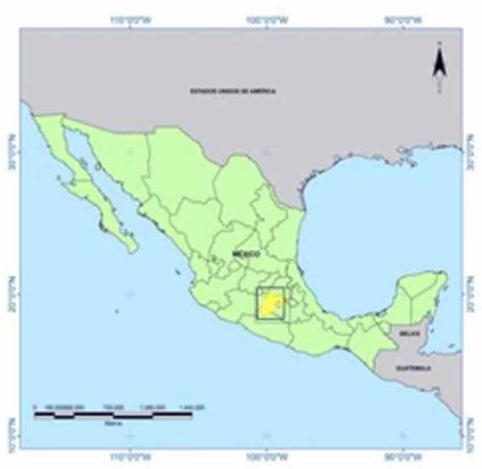

S. J. Atezcapan, Edo. Méx.: $1860 \mathrm{~m}$ Bosque tropical caducifolio

El cerrillo, Edo. Méx.: $\underline{2632} \mathrm{~m}$ Pastizal

La Cima, D. F.: $\underline{3016} \mathrm{~m}$ Bosque de Pino-Oyamel

Buenavista, Edo. Méx.: $\underline{3200 \mathrm{~m}}$ Bosque de Pino 
a)

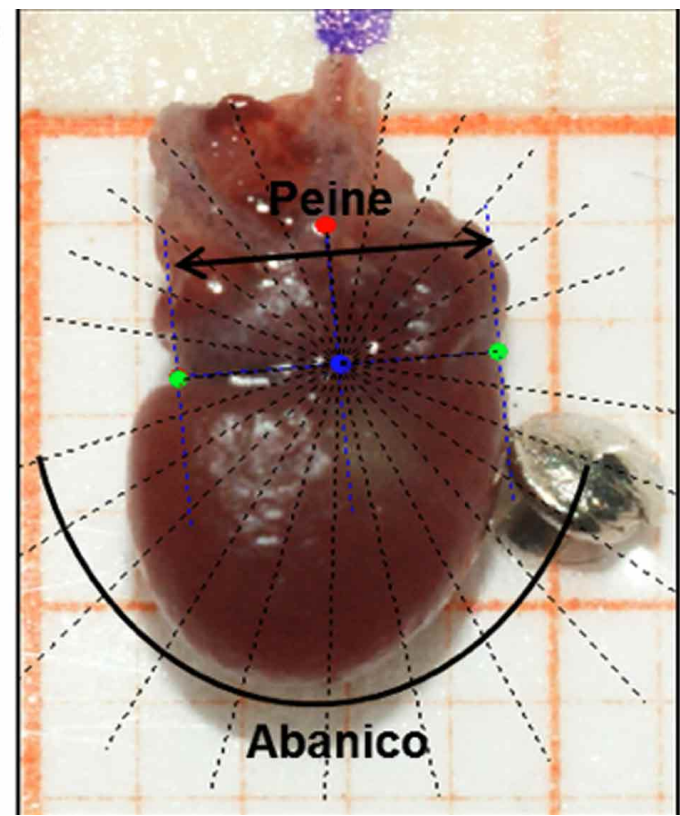

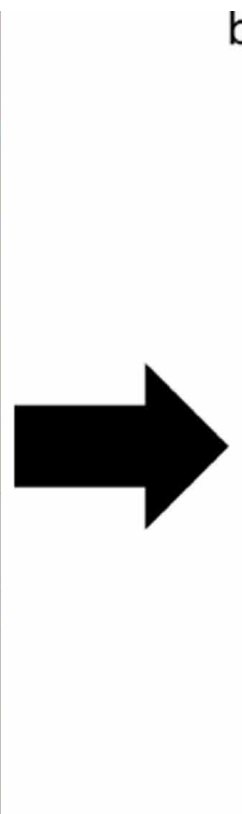
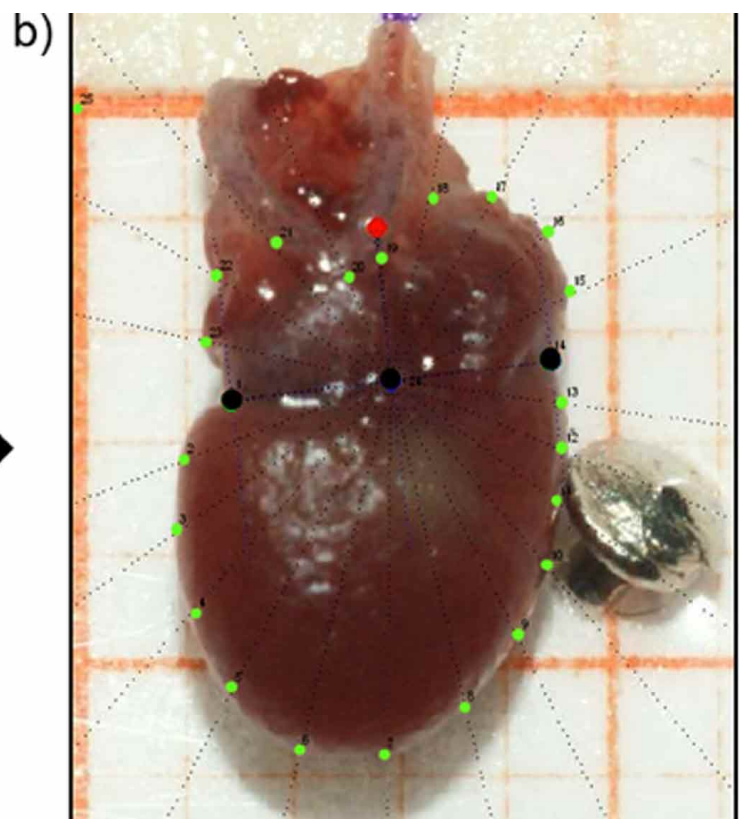

Fig. 2. a) Ubicación de peines y abanicos, b) Digitalización de landmarks (puntos negros) y semilandmarks (puntos verdes).

Una vez que se colocaron los peines y abanicos, se construyó el archivo TPS (Thin Plate Spline) en el módulo tpsUtil y éste se utilizó para digitalizar los landmarks y semilandmarks en el módulo tpsDig, ambos de la serie TPS (Rohlf, 2010). Para eliminar la variación en las configuraciones de las marcas de cada población, se realizó un método de superimposición de análisis generalizado de procrustes (GLS, por sus siglas en inglés) para eliminar los efectos de translación, rotación y escala. Posteriormente, se realizó un Análisis de Componentes Principales (ACP) y, para discriminar con respecto a la forma del corazón y clasificar correctamente a los individuos de cada población se realizó un Análisis de Variables Canónicas (AVC), estos análisis se desarrollaron con el programa Paleontological statistics (PAST; Hammer et al., 2001). Por último, para visualizar el grado de deformación de cada población mediante placas delgadas se realizó un análisis de Thin Plate Spline con el módulo de PCAGen del programa IMP (Sheets, 2001).

\section{RESULTADOS}

En el análisis de componentes principales (ACP), el CP1 y el CP2 explican 70\% de la variación morfológica, lo que indica que hay una alta diferenciación en la forma de los corazones con respecto a la altitud. El CP1 explica el mayor porcentaje de variación con relación a la forma del corazón ( $53 \%$ de la varianza total). La placa fina de defor- mación (TPS) muestra que esta variación se encuentra asociada con el ensanchamiento de los atrios y una ligera reducción del ventrículo. En el CP2 (17\% de la varianza total), la deformación de la gradilla indica un ensanchamiento en la parte media izquierda del ventrículo y una ligera ampliación en la unión de los atrios.

Posteriormente se realizó un análisis de Variables Canónicas, para clasificar a los individuos de acuerdo a su agrupación morfológica, para ello se utilizaron los primeros cuatro componentes, ya que son los que indican el mayor porcentaje de variación entre las distancias de los semilandmarks para cada población. Los resultados de este análisis mostraron la formación de dos grupos significativamente definidos (Wilk's $1=0,3, \mathrm{df}=12, \mathrm{~F}=3,34, \mathrm{p}=0,00$; Fig. 3), uno que conjunta a las poblaciones de baja altitud (San Juan Atezcapan, $1860 \mathrm{~m}$ y El Cerrillo, $2632 \mathrm{~m}$ ) y otro con las poblaciones que habitan en altitudes mayores (La Cima, $3018 \mathrm{~m}$ y Buenavista, $3216 \mathrm{~m}$ ). A su vez, las distancias de Mahalanobis confirman la variación de la forma del corazón, lo que indica que la mayor distancia se encuentra entre las poblaciones que habitan zonas de mayor y menor altitud.

A partir de estos resultados y para definir las adaptaciones anatómicas del corazón, se compararon las placas finas de deformación para cada una de las poblaciones y se observó que en la localidad de San Juan Atezcapan (1860 m) hubo una reducción en las aurículas así como en la parte media del ventrículo. Esta tendencia en la morfología del corazón también se presentó en la población de El Cerrillo 


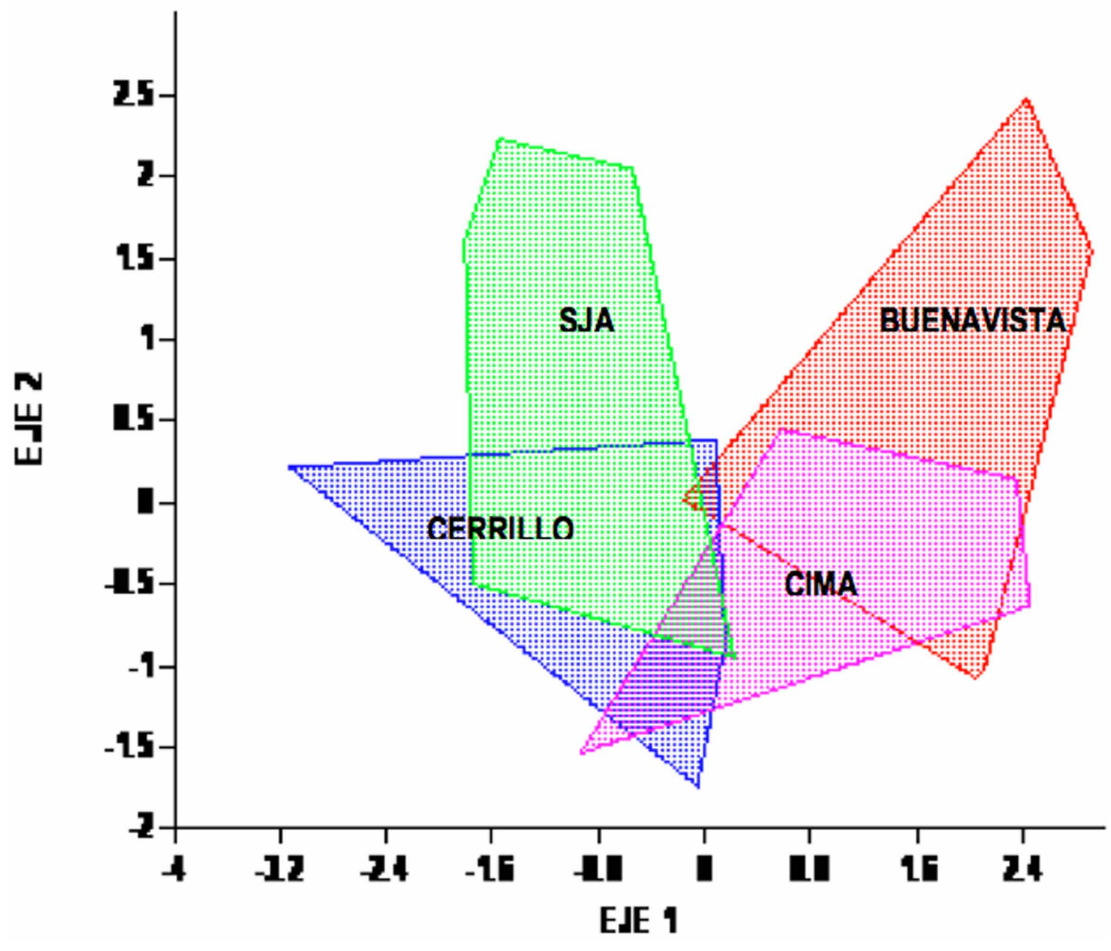

Fig. 3. Análisis de Variables Canónicas de la vista ventral del corazón con las poblaciones analizadas.

(2632 $\mathrm{m})$, pero con un ensanchamiento de la parte media derecha del ventrículo. En la población de La Cima, D.F. (3016 m), se observó una ampliación en la parte media del ventrículo y una reducción en las aurículas. Por ultimo, en la población de mayor altitud (Buenavista, $3216 \mathrm{~m}$ ), el ventrículo muestró una reducción significativa, mientras que las aurículas presentaron un mayor ensanchamiento con respecto al resto de las poblaciones (Fig. 4). a

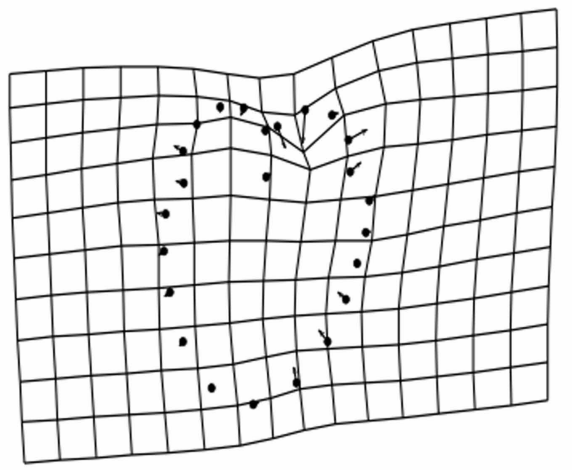

b

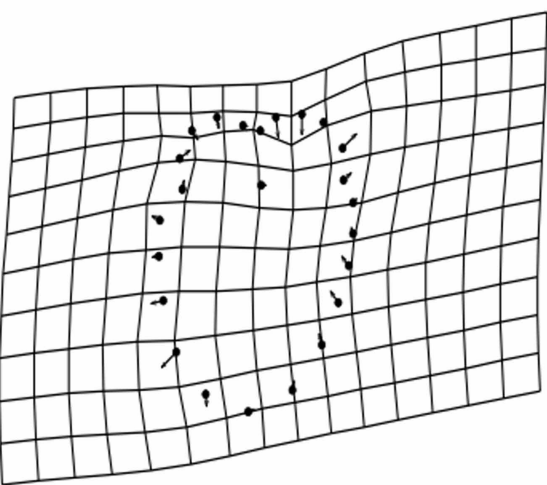

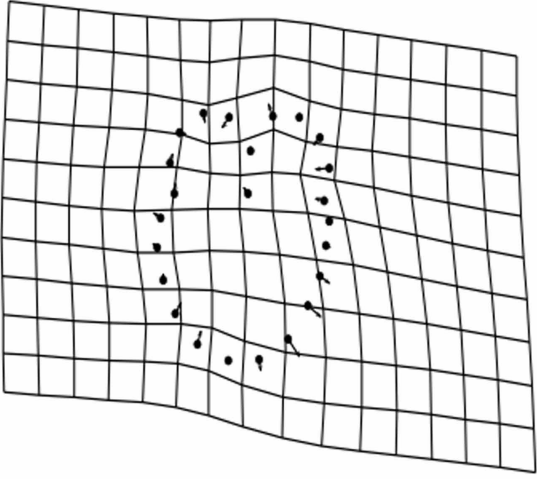

d

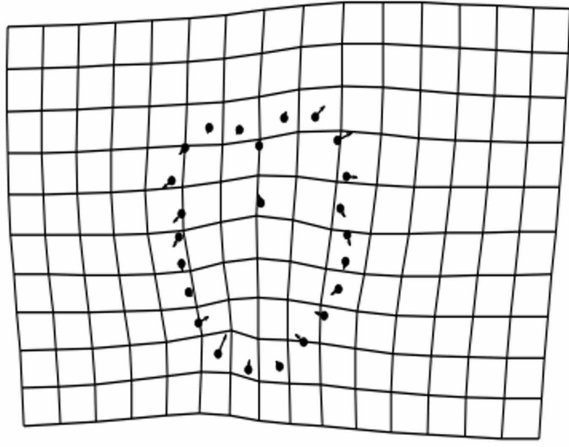

Fig. 4. Gradillas de deformación para cada una de las localidades analizadas a) San Juan Atezcapan $(1860 \mathrm{~m})$, b) El Cerrillo (2632 m), c) La Cima (3016 m), d) Buenavista (3200 $\mathrm{m})$. 


\section{DISCUSIÓN}

Los resultados del presente estudio, indican una variación estadísticamente significativa en la morfología del corazón de las poblaciones de Hyla plicata analizadas en el presente estudio. Aquellas que se encuentran en un intervalo de altitud entre $1800 \mathrm{~m}$ y $2600 \mathrm{~m}$, no parecen ser desafiados por las condiciones que se presentan en estas altitudes. Sin embargo, las poblaciones que habitan en altitudes de más de $3000 \mathrm{~m}$, presentan cambios en alguna de las regiones del corazón. Este ambiente "extremo", se distingue por presentar niveles bajos de oxigeno, lo que promueve que los organismos estén sometidos a condiciones de hipoxia (Eckert, 2006) así como a temperaturas bajas que definitivamente afectan la fisiología y el rendimiento en estos vertebrados ectotermos (Navas, 2003). Para que estas poblaciones pudieran habitar en ambientes constantes de hipoxia y baja temperatura, pasaron por un proceso de adaptación que moldeo las estrategias de historia de vida de estos organismos para colonizar el ambiente de alta montaña.

De las principales adaptaciones a la altura se caracterizan aquellas que en su conjunto facilitan el transporte de oxígeno a los tejidos del cuerpo (Snyder, 1978). Además, el cambio en la morfología del corazón de los organismos que viven a mayor altitud, está ocasionado por las sobrecarga de presión y volumen de la sangre, lo que ocasiona un mecanismo de hipertrofia adaptativa. La hipertrofia es la respuesta de los tejidos y órganos ante un aumento de estrés, ya sea por la demanda funcional, estimulación hormonal especifica o por un incremento en el trabajo físico; este mecanismo de adaptación, ayuda a que la carga de trabajo del bombeo de sangre en el corazón de poblaciones de gran altitud, este complementada con una mayor masa de componentes celulares, como son los miocitos (De la Serna, 2010; Kumar et al., 2005).

Aunado a lo anterior, el miocardio es uno de los tejidos que más depende del catabolismo aeróbico y tiene una necesidad urgente por recibir oxígeno, debido a que realiza un trabajo intenso y persistente; por ende sus células dependen especialmente de un aporte constante de oxígeno. Por lo que se sugiere que al tener un corazón más ensanchado en altitudes superiores donde los niveles de oxígeno son pobres, la cavidad ventricular aumenta y puede recibir una mayor cantidad de sangre, lo que hará que el volumen sistólico se intensifique y el aporte de oxígeno a los tejidos sea rápido y constante, además, logrará con estas características abastecer sus propias exigencias de oxígeno (Hill et al.; Moyes \& Schulte; Jackson).

Las adaptaciones tanto morfológicas como fisioló- gicas en el corazón, hacen posible un bombeo más eficaz de la sangre así como una renovación intensa del oxígeno a los diferentes tejidos del cuerpo, lo que mitiga la falta de este vital elemento en ambientes de gran altitud (Scott et al.; Alerstam). Diferentes autores han registrado esta adaptación en otros vertebrados (reptiles, aves y mamíferos) que habitan en gran altitud (Ruiz-Valencia; Liao et al., 2010; Scott et al.; Hill et al.; Campbell et al.; Alerstam) además, de otros ajustes fisiológicos que aumentan la capacidad de transportar oxígeno a la sangre (Scott et al.; Moyes \& Schulte; Jackson). La mayoría de estas investigaciones se han basado en análisis controlados de laboratorio que solo reflejan un aproximado de lo que ocurre en ambientes de gran altura y con otro tipo de técnicas, por lo que la presente investigación es una de las primeras en mostrar las adaptaciones morfológicas en el corazón de los anfibios en ambientes naturales de gran altitud, con análisis estadísticos multivariados que permiten evaluar los cambios en la morfología del órgano.

Finalmente, los anfibios a pesar de ser animales ectodermos ocupan ambientes similares que los endotermos, por lo que muestran ciertas adaptaciones que tienen estos vertebrados, aunque al parecer se basan en diferentes mecanismos (evolución convergente), estas características adaptativas ocurren porque ambos grupos están sujetos a presiones selectivas similares.

AGRADECIMIENTOS. A la Dra. Ruth Moreno Barajas, por su colaboración en la parte de morfometría geométrica, al Consejo Nacional de Ciencia y Tecnología por el otorgamiento de la beca nacional $\mathrm{N}^{\circ} 238370$ y al proyecto UAEMEX-2463 por el financiamiento a este proyecto.

RIOS-RODAS, L.; RODRÍGUEZ-ROMERO, F. J.; VELÁZQUEZRODRÍGUEZ, A. S. \& HERNÁNDEZ-FRANYUTTI, A. A. Geometric Morphometric of the heart in Hyla plicata through an altitudinal gradient in the Mexican Neovolcanic Axe. Int. J. Morphol., 31(3):905-910, 2013.

SUMMARY: It has been reported that the air environment is less problematic for organisms in relation to hypoxia except for special circumstances, such as exposure to high altitudes or burrows. The high altitude environment is challenging in different ways, since there are biotic and abiotic factors may interact to determine the distribution and survival of organisms. For these aspects, biology is based on the ecomorphology, area that helps to define the morphological differences with respect to habitat change and geometric morphometrics to analyze statistically those changes. In the present study, we evaluated different populations of the frog Hyla plicata is a species that are distributed at higher elevations in Mexico (1500 $\mathrm{m}$ to $3700 \mathrm{~m}$ ). For the purpose of this study was to analyze the morphology in Hyla plicata heart through an altitudinal gradient. The organisms were collected at four sites, one was located in La Cima (Distrito Federal to $3016 \mathrm{~m}$ ), and three in the Estado of Mexico: Buenavista (3200 m), El Cerrillo (2632 m) and San Juan Atezcapan (1860 m). The organisms collected were dissected to extract the heart for the purpose of scan them through photographs, and analyzed with geometric points and thus evaluate the 
shape of the organ. The results indicate that higher altitude locations have enlarged atria and ventricles, indicating that this structure evolved and adapted to effect a more efficient pumping of blood and then direct it to different body tissues. This adaptation in the morphology of the heart, allowed to adapt to Hyla plicata in high altitude environments as seen in other vertebrates that inhabit higher elevations.

KEY WORDS: Geometric morphometric; Adaptation; Altitudinal gradient; Hyla plicata; Heart.

\section{REFERENCIAS BIBLIOGRÁFICAS}

Arlerstam, T. Bird Migration. Cambrige, Cambrige University Press, 1990.

Campbell, N. A.; Mitchell L. G. \& Reece, J. B. Biología: Conceptos y relaciones. México D. F., Pearson Educación, 2001.

De la Serna, F. Hipertrofia ventricular, Remodelación, Apoptosis en: Insuficiencia cardiaca crónica. 3a ed. Buenos Aires, Federación Argentina de Cardiología, 2010.

Duellman, W. E. The hilyd frogs of frogs of Middle America. Vols. 1 y 2. Monographs of the Museum of Natural History. Lawrence, Kansas, University of Kansas, 1970.

Eckert, R. Fisiología animal, Mecanismos y adaptaciones. Madrid, Interamericana, 2006.

Hammer, O.; Harper, D. A. T. \& Ryan, P. D. PAST. Paleontological Statistic Sofware Package for Education and Data Analysis. Paleontol. Electron., 4(1):9, 2001. Disponible en: http://palaeo-electronica.org/ 2001_1/past/issue1_01.htm.

Hill, R. W. Fisiología Animal Comparada: un enfoque ambiental. Barcelona, Reverte, 2007.

Hill, R. W.; Wyse G. A. \& Anderson M. Animal Physiologic. Madrid, Médica Panamericana, 2008.

Hillman, S. S.; Withers, P. C.; Drewes, R. C. \& Hillyard, S. D. Ecological and Environmental Physiology of Amphibians. Oxford University Press Inc., 2009.

Hutchison, V. H. Physiological Ecology of the Telmatobiid frogs of Lake Titicaca. Nat. Geogr. Soc. Res. Rep., 14:357-61, 1982.

Hutchison, V. H.; Haines, H. B. \& Engbretson, G. Aquatic Life at High Altitude: Respiratory Adaptations in the Lake Titicaca frog, Telmatobius culeus. Respir. Physiol., 27(1):115-29, 1976.

Jackson, D. C. Temperature and hypoxia in ectothermic tetrapods. J. Therm. Biol., 32(3):125-33, 2007.

Kumar, V.; Abbas, A. K. \& Faustos, N. Patología estructural y funcional. $7^{\mathrm{a}}$ ed. Madrid, Elsevier, 2005.

Liao, J.; Wang, L.; Zhao, L. \& Liu, N. Effects of environmental factors on organ mass of midday gerbil (Meriones meridianus Pallas, 1773). Mamm. Biol., 75(5):381-8, 2010.

Méndez, M. A.; Soto, E. R.; Correa, C. C.; Veloso, A.; Vergara, E.; Salaverry, M. \& Iturra, P. Morphological and genetic differentiation among Chilean populations of Bufo spinulosus (Anura: Bufonidae). Rev. Chil. Hist. Nat., 77:559-67, 2004.
Marqués, J. I.; Leito, D. T.; Spainka, P. H.; Testerink, J. P.; Jaspers. T. R.; Witte, F.; Berg, S. \& Bagowski, P. C. Transcriptome analysis of the response to chronic constant hypoxia in zebrafish hearts. J. Comp. Physiol. B, 178(1):77-92, 2008.

Moyes D. C. \& Schulte, P. M. Principios de Fisiología Animal. Madrid, Pearson Educación S. A., 2008.

Navas, C. A. Herpetological diversity along Andean elevational gradients: links with physiological ecology and evolutionary physiology. Comp. Biochem. Physiol. A Mol. Integr. Physiol., 133(3):469-85, 2003.

Rohlf, F. J. Software tpsDig, versión 2.15 @. Department of Ecology and Evolution, State University of New York at Stony Brook, NY 117945245, 2010. Disponible en: http://www.life.bio.sunysb.edu/morpho/ index.html

Ruiz-Valencia, M. M. Morfometría externa del corazón en dos especies hermanas del grupo Scalaris, con diferente modo reproductor en diferentes altitudes. Tesis de Licenciatura, Universidad Autónoma del Estado de México, 2010.

Ruiz, G.; Rosenmann, M. \& Veloso, A. Respiratory and hematological adaptations to high altitude in Telmatobius frogs from the Chilean andes. Comp. Biochem. Physiol. A Comp. Physiol., 76(1):109-113, 1983.

Ruiz, G.; Rosenmann, M. \& Veloso, A. Altitudinal Distribution and Blood Values in the toad, Bufo spinulosus Wiegmann. Comp. Biochem. Physiol. A Comp. Physiol., 94(4):643-6, 1989.

Scott, R. G.; Egginton, S.; Richards, J. G. \& Milsom, W. K. Evolution of muscle phenotype for extreme high altitude flight in the bar-headed goose. Proc. Biol. Sci., 276(1673):3645-53, 2009.

Sheets, H. D. Software IMP (C) (Integrated Morphometric Package) Department of Physics, Canisius College, Buffalo, NY 1408, Department of Geology State University of New York atn Buffalo, NY 14260, 2001. Disponible en: http://www.canisius.edu/ sheets/ morphsoft.html

Snyder, L. R. Genetics of hemoglobin in the deer mouse, Peromyscus maniculatus. Genetics, 89(3):511-30, 1978.

Valenzuela, A.; Alveal, K. \& Tarifeño, E. Respuestas hematológicas de Truchas (Oncorhynchus mykiss Walbaum 1792) a estrés hipóxico agudo: Serie Roja. Proceeding of the IV Symposium-Workshop of Chilean Association of Ichthyology. Guayana, 66(2):255-61, 2002.

Zelditch, M. L.; Swiderski, D.; Sheets, D. H. \& Fink, W. Geometric Morphometrics for Biologists: A Primer, New York, Academic Press, 2004.

Dirección para Correspondencia:

Biól. Liliana Rios Rodas

Facultad de Ciencias

Universidad Autónoma del Estado de México

El Cerrillo Piedras Blancas, Toluca

Estado de México. C.P. 50200.

MÉXICO

Email: ari1707@hotmail.com

Recibido: 08-05-2012

Aceptado: 22-08-2013 